\title{
High Performance Wireless Switch Protocol for IEEE 802.11 Wireless Networks
}

\author{
JENHUI CHEN* \\ Department of Computer Science and Information Engineering, Chang Gung University, Kweishan, Taoyuan, Taiwan, R.O.C. \\ AI-CHUN PANG \\ Department of Computer Science and Information Engineering, National Taiwan University, Taipei, Taiwan, R.O.C. \\ SHIANN-TSONG SHEU \\ Department of Electrical Engineering, Tamkang University, Tamsui, Taipei, Taiwan, R.O.C. \\ HSUEH-WEN TSENG \\ Department of Computer Science and Information Engineering, National Taiwan University, Taipei, Taiwan, R.O.C.
}

\begin{abstract}
All mobile stations (STAs) in IEEE 802.11 infrastructure wireless local area networks (IWLAN) are coordinated by an access point (AP). Within the $2.4 \mathrm{GHz}$ unlicensed industry, science, and medicine (ISM) band defined in the IEEE $802.112 .4 \mathrm{GHz}$ physical layer (PHY) specifications, three channels are available for concurrently transferring data packets at the coverage area of an AP. In most of small/medium enterprises or home environments, an AP with one selected channel is sufficient for covering whole service area, but this implies that the radio resources for the remaining two channels are wasted. In order to overcome the drawback, we propose a new and simple media access control (MAC) protocol, named wireless switch protocol (WSP), for increasing the throughput of IEEE 802.11 IWLAN network to support high quality multimedia traffic. This is achieved by allowing any pair of STAs in IWLAN to exchange data packets in one of other idle channels after their handshake with each other in the common channel controlled by AP. Simulation results show that the total network throughput of WSP depends on the time taken by channel switching, and on the 'Intranet' and 'Internet' traffic distribution, where the Intranet and Internet mean data transmission between STAs in IWLAN and between the STA and wired host, respectively. When all data packets are Intranet traffic and the traffic load is heavy, the ratio of Goodput for the proposed WSP to that of IEEE 802.11 standard approximates $400 \%$. In the worse case of all Internet traffic, the proposed WSP still obtains the similar throughput as that of IEEE 802.11 standard.
\end{abstract}

Keywords: ad hoc, CSMA/CA, infrastructure, MAC, switch, wireless

\section{Introduction}

With the explosive growth of wireless subscribers, wireless communication technologies play an increasingly important role in recent years, which provides users with network connectivity without being restricted by wired lines. With high data rate transmission, supporting multimedia applications may be feasible in wireless local area network (WLAN). To efficiently provide multimedia applications over WLAN, the access protocol for WLAN needs to be carefully designed especially on the problem of bandwidth consumption. Intuitively, a wireless medium access control (MAC) protocol that guarantees the quality of real-time services (such as delay bound, delay jitter, minimal bit rate, and frame loss ratio, etc.) should be more complicated than a simple contention based protocol is. Take the wired Ethernet with IEEE 802.3 carrier sense multiple access with collision detection (CSMA/CD) MAC protocol as an example. The way of supporting multimedia applications (e.g., video on demand) over the wired Ethernet is to enhance the transmission rate, not to compli-

\footnotetext{
${ }^{*}$ Corresponding author.
}

cate the IEEE 802.3 MAC protocol. This strategy defeats the 100VGanyLAN with IEEE 802.12 demand priority protocol, which provides different priorities for data frames. Based on this experience, this paper focuses on the way of improving the effective transmission rate by slightly modifying IEEE 802.11 carrier sense multiple access with collision avoidance (CSMA/CA) MAC protocol.

IEEE 802.11 WLAN supports two kinds of network operation models: Infrastructure WLAN (IWLAN) $[8,17]$ and ad hoc WLAN $[8,18,19]$. In IWLAN, the access point (AP) plays an important role of bridge between wired (e.g., Ethernet/Internet) and wireless networks, and optionally provides the polling function for time-constrained services. Since AP is the coordinator of IWLAN, all STAs in the BSS can hear it and synchronize with it (by listening periodic beacon frames), On the contrary, ad hoc WLAN is a collection of STAs, which quickly forms the temporary data exchange network without the centralized administration. In this paper, we only discuss the enhanced approach for the pervasive IWLAN with AP(s).

The unlicensed nature of ISM (Industrial, Science, and Medical) band makes IEEE 802.11 IWLAN standard extremely attractive for customers or operators. In the Direct 
Sequence Spread Spectrum (DSSS) physical layer (PHY) specification [8], total radio resources of 83.5 MHz bandwidth are spreaded and divided into 14 partially overlapping channels. However, using all channels to transmit data at a same location will cause severe electromagnetic wave interferences that degrade the transmission quality. Therefore, the IEEE $802.11 \mathrm{~b}$ standard suggests that at least $25 \mathrm{MHz}$ or $30 \mathrm{MHz}$ guard band should be maintained between any two overlapping WLANs. Hence, the standard points out that three available channels (centered at $2.412 \mathrm{GHz}, 2.437 \mathrm{GHz}$, and 2.462 $\mathrm{GHz}$ in North American or centered at $2.412 \mathrm{GHz}, 2.442 \mathrm{GHz}$, and $2.472 \mathrm{GHz}$ in Europe excepting Spain and France) can be used to delivery data frames in the same area at the same time. In other words, since the highest channel data rate is 11 $\mathrm{Mb} / \mathrm{s}$ in IEEE $802.11 \mathrm{~b}$ and $54 \mathrm{Mb} / \mathrm{s}$ in IEEE $802.11 \mathrm{~g} 2.4 \mathrm{GHz}$ PHY draft [9], the aggregated network bandwidth can be up to $33 \mathrm{Mb} / \mathrm{s}$ in IEEE $802.11 \mathrm{~b}$ and $162 \mathrm{Mb} / \mathrm{s}$ in IEEE $802.11 \mathrm{~g}$ draft [11].

In general, for the ordinary small/medium enterprises or home environment, one IEEE 802.11b AP with adaptive rate control is sufficient for covering the service area; this implies that only one channel is used and the other two channels (with total capacity $22 \mathrm{Mb} / \mathrm{s}$ ) are useless according to IEEE $802.11 \mathrm{~b}$ Specification [9]. In order to effectively utilize the available radio resources, we propose a novel and simple CSMA based protocol named as Wireless Switch Protocol (WSP) for raising the aggregated throughput in IEEE 802.11 IWLAN. The proposed WSP allows any pair of STAs in IWLAN to exchange frames in another available channel after handshaking between the two STAs is performed in a pre-specified channel controlled by AP. For simplicity, the pre-specified channel is named as the common channel and the other channels are named as the data channels throughout this paper. With WSP, a higher bandwidth capacity can be provided to meet the quality requirement for multimedia services over IWLAN.

Authors in the papers [23,24] had proposed possible solutions for ad hoc WLAN by adopting dual transceivers for the STA where one transceiver is fixed in a dedicated common channel for channel contention, and the other transceiver is tunable among all data channels for data transmission. Nevertheless, the requirement for dual-transceiver STAs not only increases the implementation cost but also becomes impractical for current WLAN cards. In this paper, the proposed WSP approach can achieve multi-channel and parallel transmissions over ordinary IEEE 802.11 IWLANs under the constrain of single transceiver (one transmitter and one receiver) for each STA.

The rest of this paper is organized as follows. In Section 2, we describe the basic operation of IWLAN and how our proposed wireless switch protocol (WSP) works in IEEE 802.11 IWLAN. The analytical model is given in Section 3 to investigate the performance of WSP, which is validated against the simulation experiments. In Section 4, we describe the simulation models and compare the performance of WSP with the IEEE 802.11 MAC protocol under different system parameters. Finally, conclusions and remarks are given in Section 5.

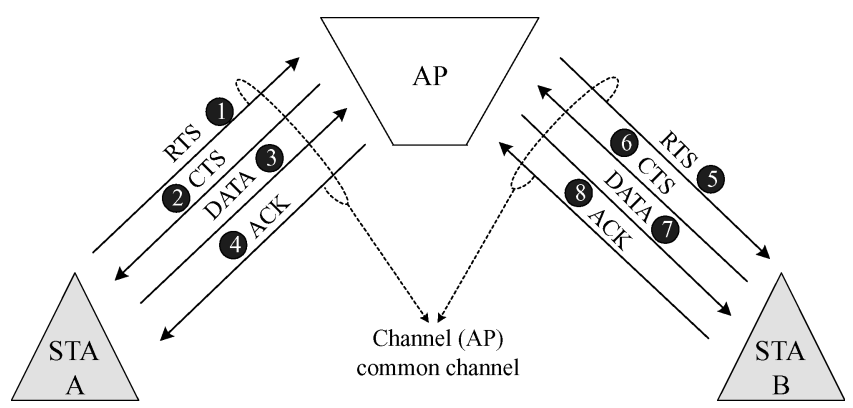

Figure 1. An illustration of data transfer from STA A to STA B via AP in IEEE 802.11 MAC protocol with RTS/CTS handshaking.

\section{The wireless switch protocol (WSP)}

This section elaborates on basic operations of IWLAN and presents our wireless switch protocol (WSP) based on IWLAN environment.

\subsection{An overview of the IWLAN}

The IEEE 802.11 MAC protocol includes a distributed coordination function (DCF) that employs CSMA/CA as the basic channel access/contention protocol for asynchronous data transmissions. With the restriction on one transceiver per wireless module, the IEEE 802.11 MAC protocol is designed to operate in a single channel (i.e., to occupy a single channel for data transmission/reception). The coverage area of a channel logically forms a basic service set (BSS). In DCF, the RTS/CTS (Request-to-Send/Clear-to-Send) handshaking between STAs and AP is used to achieve the following objectives: to solve the hidden-terminal problem, to reduce the bandwidth wastage and to provide virtual carrier sense for power saving. In the handshaking process, the originating STA (i.e., the STA originates data transmission) first issues the RTS control frame instead of the data frame. Since the frame size for RTS control frame is much smaller than that of the data frame, bandwidth wastage can be reduced if the collision occurs. Figure 1 shows an example of RTS/CTS handshaking. When STA A would like to transmit data to another STA, say $\mathbf{B}$, the RTS control frame is first issued from STA A to AP (see Step 1 in figure 1). Then STA A waits for the CTS control frame replied from AP (see Step 2 in figure 1). After executing Steps 1 and 2, all STAs in the BSS are aware of the channel occupancy and the occupancy duration for the coming access of STA A. Therefore STA A can safely send data frames and receive the acknowledgement (ACK) replied from AP (see Steps 3 and 4 in figure 1). Following the data transmission between SAT A and AP, the AP forwards the received data frame to STA B. Similar handshaking process can be found between AP and STA B (see Steps 5-8 in figure 1).

In this example, AP plays the role of relaying node between the source (STA A) and the destination (STA B), which results in several disadvantages described as follows. (1) More channel bandwidth is consumed than that for direct communication between two STAs. (2) Additional contentions and collisions 
are generated and the average access delay is increased. (3) AP needs extra buffer spaces for transmitting/receiving these data frames. (4) These data frames may suffer from a long buffer delay. (5) The data frames destined to wired hosts (denoted as Internet traffic) will be blocked by the data frames destined to neighboring wireless hosts (denoted as Intranet traffic) in the same BSS. From the above discussions, the network throughput can be significantly increased if two STAs in the same BSS are permitted to exchange data frames via another data channel that is different from the common channel used by AP. In other words, Intranet and Internet traffic can be simultaneously served via two separate channels and thus frame contentions and collisions can be further reduced. This study develops the wireless switch protocol (WSP) for multichannel IWLAN environment. Two kinds of WSPs, named complex WSP (CWSP) and simple WSP (SWSP), are proposed for outdoor IWLAN and indoor IWLAN, respectively. CWSP is designed for solving the potential hidden terminal problem in large-scale range and SWSP is used for a relatively pure environment where every STA can hear with each other (e.g., in the home networking). In the following subsections, we will elaborate on the design of CWSP and SWSP in detail.

\subsection{The complex wireless switch protocol (CWSP)}

This section describes our CWSP for outdoor IWLAN environment. In CWSP, if one STA would like to send Intranet frames to another STA via an idle data channel, both of them need to tune to the same channel at the same time for fulfilling the requirement of one transceiver per STA. As the coordinator of a BSS in IWLAN, AP is used to perform channel allocation for data frame transmission between STAs. Figure 2 shows the message flow of CWSP for direct data transfer between two neighboring STAs (i.e., STA A and STA B). In figure 2, the sender (i.e., STA A) first occupies the common channel based on the CSMA/CA protocol, and communicates with AP via M-RTS1 (Multi-channel RTS Type 1) control frame to request data channel occupancy (see Step 1 in figure 2). The frame format of M-RTS1 control frame, as shown in figure 3, is similar to that of standard RTS frame in addition to the data length

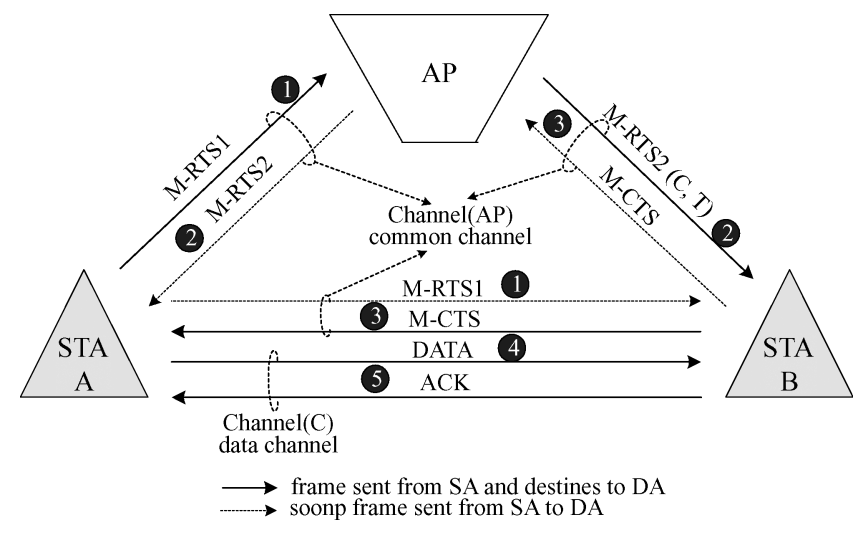

Figure 2. An illustration of direct data transfer from STA A to STA B bypassing AP in proposed CWSP. field indicating the requested duration for data channel occupancy. We note that the AP receives M-RTS1 control frame since the M-RTS1 is sent by using broadcast scheme. Upon receipt of the M-RTS1 control frame, AP recognizes the receiver (i.e., STA B) located in the same BSS as STA A, and issues a M-RTS2 (Multi-channel RTS Type 2) control frame to both STAs A and B. The M-RTS2 control frame carries the assigned data channel, say channel $C$, and the timestamp, say time $T$, of the allocated time period. ${ }^{1}$ We emphasize that when STA B detects the M-RTS1 frame, it will look forward to receiving the subsequent M-RTS2 with data channel $C$ and time stamp $T$ information sent from AP. When STA B receives the expected M-RTS2, it replies an M-CTS (with the destination address (DA) field = address of STA A) back to STA A directly (see Step 3 in figure 2). Note that AP also detects the M-CTS control frame sent from STA $\mathbf{B}$ and then updates its channel occupancy table according to the data length field in the M-RTS1 frame. After then, STAs A and B tune to data channel $C$ at the assigned time $T$ to perform data transfer. Finally, STA B replies the standard ACK (acknowledgement) control frame when the complete data frame sent from STA A has been received.

The formats of three additional control frames (M-RTS1, M-RTS2, and M-CTS) are illustrated in figure 3. In CWSP, the duration fields of M-RTS1, M-RTS2, and M-CTS are set as M-RTS1 + SIFS + M-RTS2 + SIFS + M-CTS, M-RTS2 + SIFS + M-CTS, and 0, respectively. The DCF inter-frame space (DIFS), the mobile STAs that intend to transmit the data frames exercise the backoff procedure to contend for channel occupancy. The short inter-frame space (SIFS) is the minimum gap between two successive transmitted frames. These durations are used to make sure that other STAs in the same BSS will not disturb the three-party handshaking and the corresponding values of them are shown in figure 4.

\subsection{Challenges in CWSP}

Based on the above discussions, CWSP is a simple solution for enhancing channel utilization in IWLAN. However, there are several challenges needed to be solved before obtaining the network throughput gain. These challenges are presented as follows.

1. How to indicate that a data frame belongs to Internet or Intranet traffic?

2. Due to the existing hidden-nodes in IWLAN [7,22], how to distinguish the case that sender and receiver are hidden to each other?

3. If the handshaking fails due to the radio interference, how to avoid the unnecessary bandwidth wastage?

4. How to maintain the data frame sequence between sender and receiver?

${ }^{1}$ In this paper, we use a simple channel assignment with the linear search algorithm. The channel assignment rule is based on first-fit search; that is, the channel with the earliest free time and without transceiver conflict in the sender and receiver is selected. 


\begin{tabular}{|c|c|c|c|c|c|c|c|c|}
\hline Type & Sub Type & & & & & & & \\
\hline 01 & 0000 & $\begin{array}{c}\text { Frame } \\
\text { Control }\end{array}$ & Duration & DA & SA & $\begin{array}{c}\text { Data } \\
\text { Length }\end{array}$ & $\mathrm{FCS}$ & M-RTS1 \\
\hline 01 & 0001 & $\begin{array}{c}\text { Frame } \\
\text { Control }\end{array}$ & Duration & DA & SA & Channel & Time Stamp & FCS \\
\hline 01 & 0010 & $\begin{array}{c}\text { Frame } \\
\text { Control }\end{array}$ & Duration & DA & FCS & M-CTS & & \\
\hline
\end{tabular}

Figure 3. The formats of CWSP control frames.

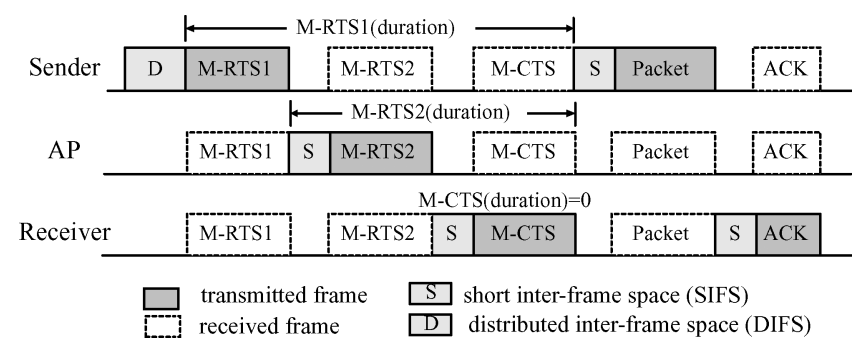

Figure 4. The durations of control frames in CWSP.

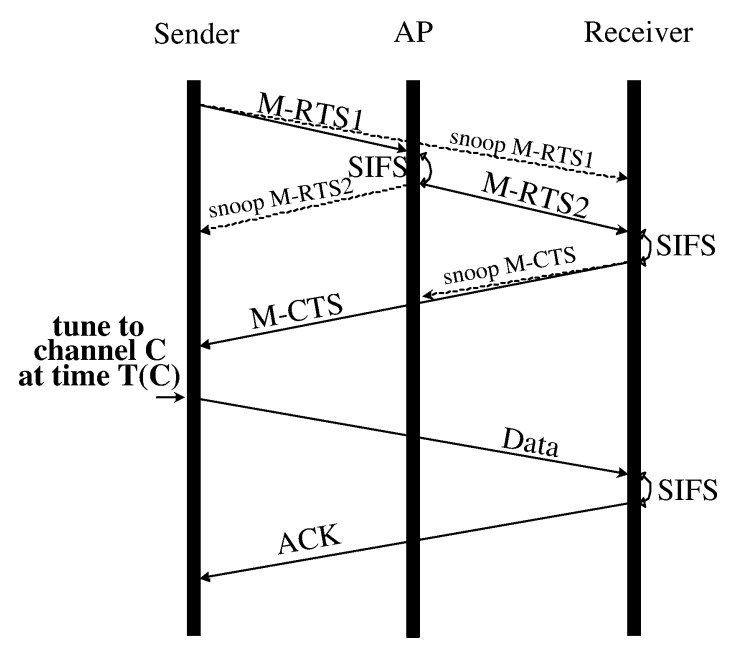

Figure 5. The normal frame sequence of CWSP.

The first challenge can be resolved by maintaining a status table in AP, which records the status for all associated STAs. Upon receipt of a new M-RTS1 control frame issued from a STA, say A, AP will check the status table to decide whether the DA is one of STAs in the same BSS. If so, AP transmits the M-RTS2 control frame to the receiving STA, say B. In this case, the message flow for control frame transmission is similar to that in figure 4 . That is, M-RTS1(B,A, $m) \rightarrow \mathrm{M}$ $\mathrm{RTS} 2(\mathrm{~B}, \mathrm{~A}, C, T) \rightarrow \mathrm{M}-\mathrm{CTS}(\mathrm{A}, \mathrm{B}) \rightarrow \operatorname{Data}(\mathrm{B}, \mathrm{A}) \rightarrow \mathrm{ACK}(\mathrm{A})$, where $m=\mathrm{PHY}_{\text {header }}+\mathrm{MAC}_{\text {header }}+$ data_length represents the exact length of transmitted data frame, $C$ is the selected channel ID, and $T$ is the starting time of data frame transmission. The detailed frame sequence is shown in figure 5. Otherwise, if the DA is not in BSS, or the transmitting and receiving STAs are hidden to each other, the AP replies a standard CTS control frame back to the sending STA to turn off the wireless switch mode. Then STA A has to transmit the data frame to STA B indirectly via AP as in the IEEE 802.11 standard. In

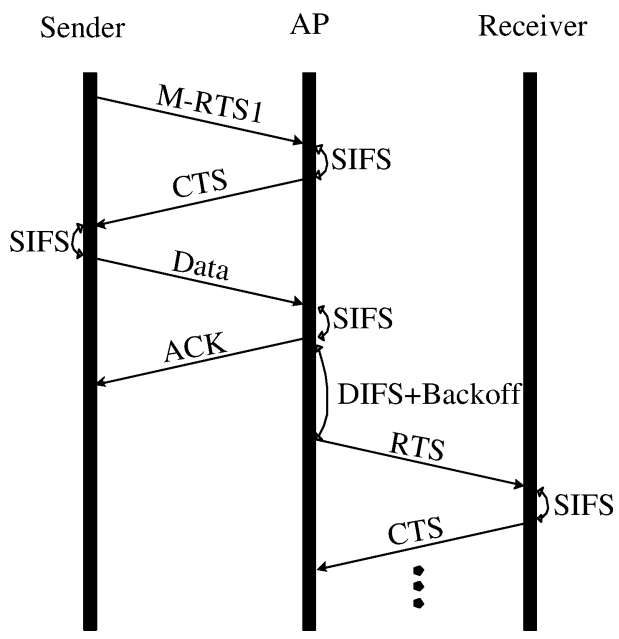

Figure 6. The frame sequence of CWSP when AP plays the role of relay or deals with Internet traffic.

this case, the transmitted control frame sequence becomes M$\mathrm{RTS} 1(\mathrm{~B}, \mathrm{~A}, m) \rightarrow \mathrm{CTS}(\mathrm{A}) \rightarrow \operatorname{Data}(\mathrm{AP}, \mathrm{A}) \rightarrow \mathrm{ACK}(\mathrm{A})$. The corresponding frame sequence is shown in figure 6 . We note that the duration field of CTS(A) is set as SIFS $+m+$ SIFS + ACK to inhibit other STAs's transmissions, which may degrade the system performance as Internet traffic occurs. To overcome this problem, a cache mechanism is used in every STA. That is, each STA needs to maintain a status table, which records the transmission mode (i.e., direct or indirect data transfer) for every communicated stations. If source STA receives the unexpected CTS control frame, it recognizes that the MAC address of the destination may belong to a wired host or that the destination STA is out of its transmission range (this case will be discussed later). Then the transmission mode for the destination STA is logged as "Indirect", and data transfer between source and destination STAs should be carried out indirectly via AP.

The second challenge can not be resolved only by the status table in AP since the AP can not inform STAs A and $\mathbf{B}$ whether they are hidden to each other. Instead, this work should be accomplished by the STAs themselves. Recall that, in CWSP, after STA A issues the M-RTS1 control frame to STA B, the AP will generate the M-RTS2 control frame to STA B. If STA B only receives the M-RTS2 control frame and misses the MRTS1 control frame, it is aware that STA A is the hidden node. Then STA B will respond to AP via transmitting a standard CTS control frame with destination address of STA A. After then, AP passes the CTS control frame to STA A for switching 


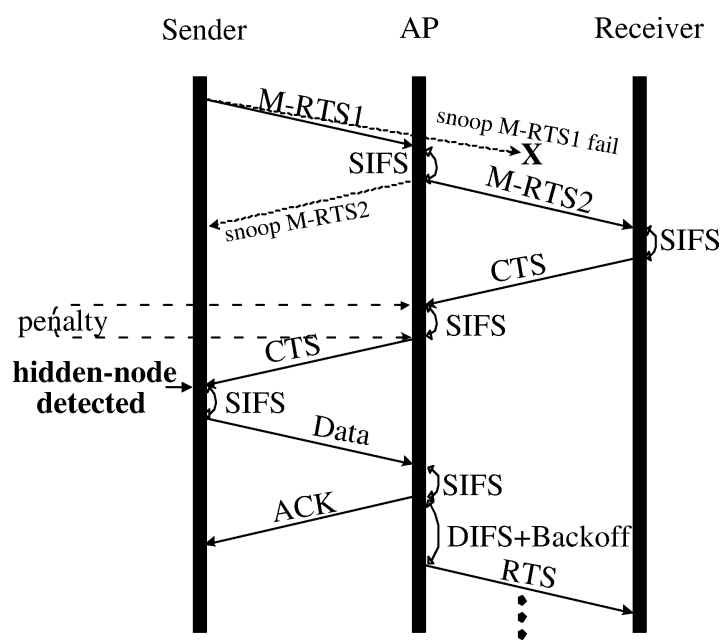

Figure 7. The frame sequence of CWSP deals with hidden node situation.

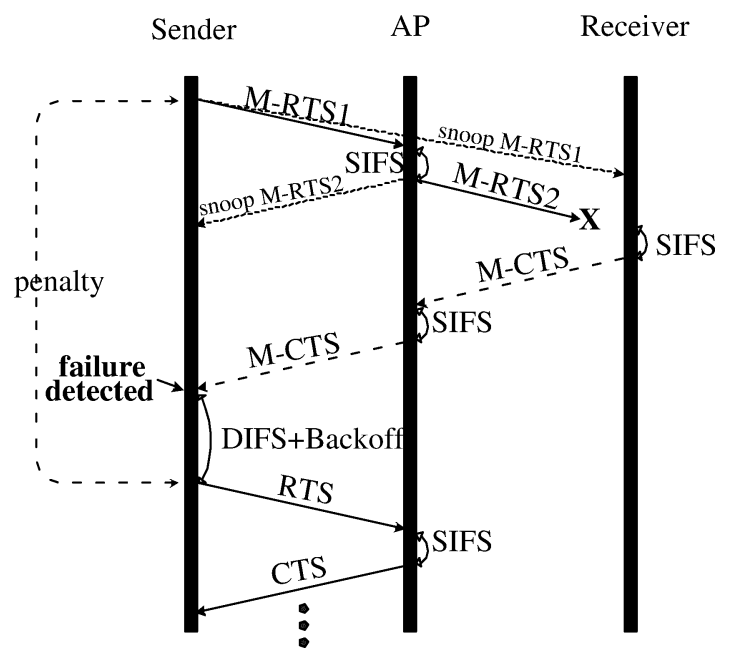

Figure 8. The frame sequence of CWSP deals with channel noise.

the transmission mode to "Indirect". The frame sequence of hidden node situation is shown in figure 7. Consequently, STA A, STA B and AP update their status tables. Furthermore, we note that when STA B receives both M-RTS1 and M-RTS2 control frames, it will reply the regular M-CTS control frame. Thus, if STA A detects the M-RTS2 control frame but does not receive the M-CTS or CTS control frame before timeout, it finds that STA $\mathbf{B}$ is a hidden node and updates its status table accordingly. The corresponding frame sequence is shown in figure 8 .

For the third challenge, if either M-RTS1 or M-CTS control frame is corrupted due to channel noise, which makes either STA B or STA A misjudges the situation, STA A or STA B could retry the multi-channel access again. From our observations, since it is hard to distinguish between hidden-node and channel noise situations, we suggest that these STAs use the standard approach to prevent from unnecessary bandwidth wastage. Consider the last challenge. If an STA is permitted to contend the channel occupancy only when it has no pending reservation, the frame sequence is guaranteed. In other words,

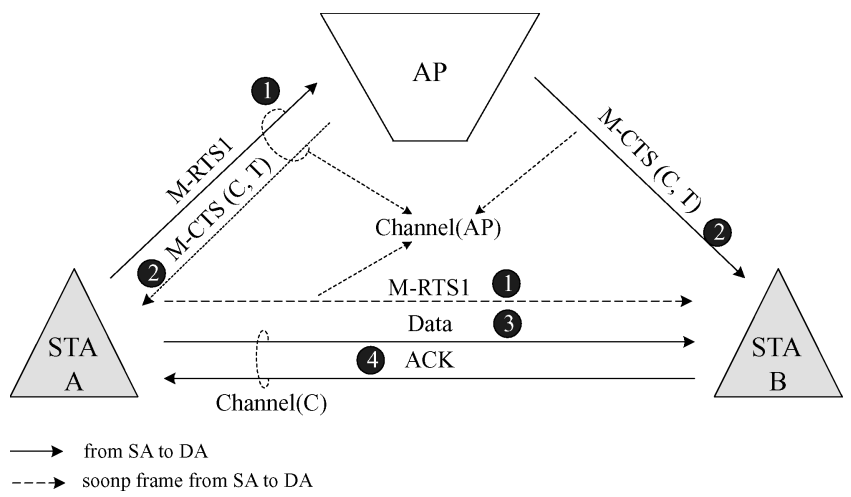

Figure 9. An illustration of direct data transfer from STA A to STA B bypassing AP in proposed SWSP.

the timing of an STA issues M-RTS1 control frame is at the moment that the previous data frame has been successfully transmitted. This approach also reduces the collision probability in the common channel and, consequently, the Internet traffic can be served more efficiently than in the existing standard.

\subsection{Simple wireless switch protocol (SWSP)}

The second WSP, named as simple wireless switch protocol (SWSP), is particularly suitable for indoor IWLAN environment such as home and office. Because of the extremely small hidden-node probability in such environment, we can assume that all STAs in a BSS are staying in a fully connected network. The normal frame sequence for SWSP is shown in figure 9 and described as follows. Similar to CWSP, an M-RTS1 control frame is sent before transmitting the data frame (see Step 1 in figure 9). When AP receives a correct M-RTS1, it decides which channel is available for this request. Then AP broadcasts the channel information via the M-CTS control frame instead of the M-RTS2 control frame used in CWSP. (Note that the frame format of M-CTS control frame used in SWSP is the same as that of the M-RTS2 control frame in CWSP.) Next, the sender and receiver will receive the M-CTS control frame from AP (see Step 2 in figure 9) and transmits the data frame in the assigned data channel.

The advantage of SWSP is that less overhead in the handshaking procedure is incurred, which improves the network throughput. However, the only disadvantage is the extra penalty resulted from hidden-node or radio-interference occurrence.

\section{System analysis}

\subsection{The analytical model}

This section proposes an analytical model to investigate the performance of the proposed CWSP and SWSP. The analysis is based on the model introduced by Tobagi and Kleinrock [22] for the CSMA protocol, and refers to $[2-4,20]$ that discuss and 


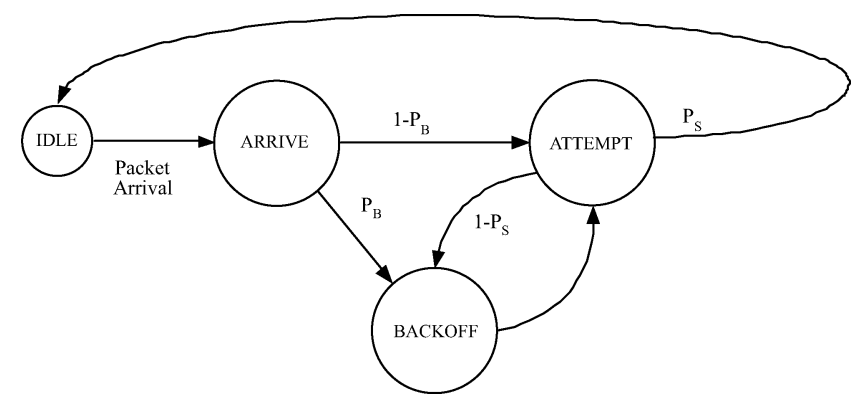

Figure 10. The state transition diagram of the WSP.

analyze the throughput of IEEE 802.11 protocol. For simplicity, the frame length is normalized as time units (slots).

Figure 10 illustrates the state transition diagram for CWSP and SWSP schemes. The diagram consists of four states: IDLE, ARRIVE, BACKOFF and ATTEMPT. Initially, the STA stays in the IDLE state. When a data frame arrives, the state transits from IDLE state to ARRIVE state. In ARRIVE state, the arriving frames are queued in the buffer of the STA. When the frame is served by the STA, the channel sensing procedure is executed. If an idle channel is found, the STA selects a random backoff time to countdown. If the STA counts to zero and senses an idle channel, the state transit from ARRIVE state to ATTEMPT state. The state transition probability is $1-P_{B}$, and the average waiting time in ARRIVE state is equal to DIFS $+W / 2$, here $W$ is the minimum backoff window size (e.g., 32 slots). Otherwise, the STA enters into BACKOFF state. The state transition probability is $P_{B}$.

In ATTEMPT state, the STA starts to perform data frame transmission. If the data frame is successfully transmitted with probability $P_{S}$, the state returns to IDLE. On the other hand, if frame transmission fails (e.g., due to the failure of RTC/CTS transmission), the state transits from ATTEMPT state to BACKOFF state with the probability of $1-P_{S}$. The derivation of $P_{S}$ is described as follows. We assume that the arrival process of frames at a STA forms the Poisson distribution with the arrival rate of $\lambda[12,15,16]$. The probability $P_{S}(\Delta t)$ that the RTS/CTS frame is successfully transmitted is given by

$$
P_{S}(\Delta t)=e^{-\Lambda \Delta t}
$$

where $\Lambda$ is the total frame arrival rate in the system. For the number $N$ of STAs, $\Lambda=N \lambda$.

When residing in BACKOFF state, the mobile station selects a random backoff time according to the backoff rule. If the minimum backoff time is selected, the state for the mobile station transits from BACKOFF state to ATTEMPT state after the end of backoff countdown.

In CWSP and SWSP, the renewal interval is defined as the time between successive renewal points. Each renewal point represents the end of successful transmission (i.e., when the sender receives the ACK control frame). A renewal interval consists of the idle and busy periods. In the idle period, the transmission medium remains idle due to backoff, DIFS interval, or no frame arrival. In the busy period, the medium is used to perform control/data frame transmission/retransmission or in collision. Based on the above discussions, the transition probability $P_{B}$ from ARRIVE state to BACKOFF state is expressed as

$$
P_{B}=\frac{\bar{B}}{\bar{I}+\bar{B}}
$$

where $\bar{I}$ and $\bar{B}$ are, respectively, the average lengths of idle and busy periods in each renewal interval. The average length $\bar{I}$ of the idle period is derived as

$$
\bar{I}=1 / \Lambda+\mathrm{DIFS}+W / 2 .
$$

Before deriving the average length of the busy period $\bar{B}$, the notations used in the derivation are introduced first. Let $T_{\mathrm{s}}$ and $T_{\mathrm{c}}$ respectively be the average times for the intervals of successful handshaking and collision occurrence. A superscript 5 for $T_{\mathrm{s}}$ and $T_{\mathrm{c}}$ denotes the 5-way handshaking involved in the transfer of a data frame in CWSP protocol. Then we can find that

$$
\left\{\begin{array}{l}
T_{\mathrm{c}}^{5}=\mathrm{M}-\mathrm{RTS} 1+\tau+\mathrm{SIFS} \\
T_{\mathrm{s}}^{5}=\mathrm{M}-\mathrm{RTS} 1+\mathrm{M}-\mathrm{RTS} 2+3 \tau+2 \mathrm{SIFS}+\mathrm{M}-\mathrm{CTS}
\end{array}\right.
$$

where $\tau$ is the propagation delay for control frame transmission. The propagation delay $\tau$ can be omitted for the data frame since the transmission time for the data frame is extremely large compared to $\tau$.

In SWSP, we use a superscript 2 for $T_{\mathrm{s}}$ and $T_{\mathrm{c}}$ to denote the 2-way handshaking. Then

$$
\left\{\begin{array}{l}
T_{\mathrm{c}}^{2}=\mathrm{M}-\mathrm{RTS} 1+\tau+\mathrm{SIFS} \\
T_{\mathrm{s}}^{2}=\mathrm{M}-\mathrm{RTS} 1+2 \tau+\mathrm{SIFS}+\mathrm{M}-\mathrm{CTS}
\end{array}\right.
$$

We use a superscript 4 for $T_{\mathrm{s}}$ and $T_{\mathrm{c}}$ to denote the IEEE 802.11 CSMA/CA 4-way handshaking. Therefore, we have

$$
\left\{\begin{array}{l}
T_{\mathrm{c}}^{4}=\mathrm{M}-\mathrm{RTS} 1+\tau+\mathrm{SIFS} \\
T_{\mathrm{s}}^{4}=\mathrm{M}-\mathrm{RTS} 1+3 \tau+3 \mathrm{SIFS}+\mathrm{CTS}+\bar{m}_{r}+\mathrm{ACK}
\end{array}\right.
$$

where $\overline{m_{r}}$ indicates the mean length of data frame with the transmission rate $r$. The performance analysis for SWSP is derived as follows. We use the probability $P_{\text {out }}$ to denote the probability that data frames will be transferred to the Internet. Thus, the average length of busy period $\overline{B^{2}}$ in SWSP can be derived as

$$
\begin{aligned}
\overline{B^{2}}= & \left(1-P_{S}\right) T_{\mathrm{c}}^{2}+P_{S}\left[P_{\text {out }} T_{\mathrm{s}}^{4}+\left(1-P_{\text {out }}\right) T_{\mathrm{s}}^{2}\right] \\
= & \text { M-RTS } 1+\tau+\operatorname{SIFS}+e^{-\Lambda \tau}\left[P _ { \text { out } } \left(\mathrm{CTS}+\tau+\bar{m}_{r}\right.\right. \\
& +2 \mathrm{SIFS}+\mathrm{ACK}-\mathrm{M}-\mathrm{CTS})+\mathrm{M}-\mathrm{CTS}+\tau] .
\end{aligned}
$$

According to (3.2), (3.3) and (3.7), the probability $P_{B}$ of SWSP (denoted as $P_{B}^{2}$ ) from ARRIVE to BACKOFF states 
can be

$$
\begin{aligned}
P_{B}^{2}= & \frac{\overline{B^{2}}}{1 / \Lambda+\mathrm{DIFS}+W / 2+\overline{B^{2}}} \\
= & \mathrm{M}-\mathrm{RTS} 1+\tau+\mathrm{SIFS}+e^{-\Lambda \tau}\left[P_{\mathrm{out}}(\mathrm{CTS}+\tau\right. \\
& \left.\left.+2 \mathrm{SIFS}+\bar{m}_{r}+\mathrm{ACK}-\mathrm{M}-\mathrm{CTS}\right)+\mathrm{M}-\mathrm{CTS}+\tau\right] \\
& \times\{1 / \Lambda+\mathrm{DIFS}+W / 2+\mathrm{M}-\mathrm{RTS} 1+\tau+\mathrm{SIFS} \\
& +e^{-\Lambda \tau}\left[P _ { \mathrm { out } } \left(\mathrm{CTS}+2 \tau+2 \mathrm{SIFS}+\bar{m}_{r}+\mathrm{ACK}\right.\right. \\
& -\mathrm{M}-\mathrm{CTS})+\mathrm{M}-\mathrm{CTS}+\tau]\}^{-1} .
\end{aligned}
$$

Similarly, the average length of busy period $\overline{B^{5}}$ in CWSP is derived as

$$
\begin{aligned}
\overline{B^{5}}= & \left(1-P_{S}\right) T_{\mathrm{c}}^{5}+P_{S}\left[P_{\text {out }} T_{\mathrm{s}}^{4}+\left(1-P_{\text {out }}\right) T_{\mathrm{s}}^{5}\right] \\
= & \text { M-RTS } 1+\tau+\mathrm{SIFS}+e^{-\Lambda \tau}\left[P_{\mathrm{out}}(\mathrm{CTS}+\mathrm{SIFS}\right. \\
& \left.+\bar{m}_{r}+\mathrm{ACK}-(\mathrm{M}-\mathrm{RTS} 2+\mathrm{M}-\mathrm{CTS})\right)+\mathrm{M}-\mathrm{RTS} 2 \\
& +2 \tau+\mathrm{SIFS}+\mathrm{M}-\mathrm{CTS}],
\end{aligned}
$$

and the probability $P_{B}^{5}$ from ARRIVE to BACKOFF states for CWSP can be

$$
\begin{aligned}
P_{B}^{5}= & \frac{\overline{B^{5}}}{1 / \Lambda+\mathrm{DIFS}+W / 2+\overline{B^{5}}} \\
= & \mathrm{M}-\mathrm{RTS} 1+\tau+\mathrm{SIFS}+e^{-\Lambda \tau}\left[P_{\mathrm{out}}(\mathrm{CTS}+\mathrm{SIFS}\right. \\
& \left.+\bar{m}_{r}+\mathrm{ACK}-(\mathrm{M}-\mathrm{RTS} 2+\mathrm{M}-\mathrm{CTS})\right)+\mathrm{M}-\mathrm{RTS} 2 \\
& +2 \tau+\mathrm{SIFS}+\mathrm{M}-\mathrm{CTS}] \times\{1 / \Lambda+\mathrm{DIFS}+W / 2 \\
& +\mathrm{M}-\mathrm{RTS} 1+\tau+\mathrm{SIFS}+e^{-\Lambda \tau}\left[P_{\text {out }}(\mathrm{CTS}+\mathrm{SIFS}\right. \\
& \left.+\bar{m}_{r}+\mathrm{ACK}-(\mathrm{M}-\mathrm{RTS} 2+\mathrm{M}-\mathrm{CTS})\right)+\mathrm{M}-\mathrm{RTS} 2 \\
& +2 \tau+\mathrm{SIFS}+\mathrm{M}-\mathrm{CTS}]\}^{-1} .
\end{aligned}
$$

\subsection{Average access delay}

Let $\bar{D}$ be the average MAC delay of a frame arrival in CWSP and SWSP. That is, $\bar{D}$ is the interval between the time that STA serves the frame and the time that the frame is successfully transmitted. Thus we have

$$
\bar{D}=P_{B}\left(\bar{B} / 2+E_{B}\right)+\left(1-P_{B}\right)\left(\mathrm{DIFS}+W / 2+E_{A}\right),
$$

where $E_{A}$ and $E_{B}$ are the average waiting times in ATTEMPT and $\mathrm{BACKOFF}$ states, respectively. From figure $6, E_{A}$ is given by

$$
\begin{aligned}
E_{A}= & P_{S}\left[P_{\text {out }} T_{\mathrm{s}}^{4}+\left(1-P_{\text {out }}\right) T_{\mathrm{s}}\right] \\
& +\left(1-P_{S}\right)\left(T_{\mathrm{c}}+E_{B}\right) .
\end{aligned}
$$

Similarly, $E_{B}$ is derived as

$$
E_{B}=\mathrm{DIFS}+\bar{W}+E_{A} .
$$

where $\bar{W}$ is the mean backoff time for a frame transmission and from [5],

$$
\bar{W}=\sum_{n=0}^{4}\left(P_{S}\left(1-P_{S}\right)^{n} 2^{n-1} W\right)+\left(1-P_{S}\right)^{5} 2^{4} W .
$$

From (3.12) and (3.13), $E_{A}$ is re-written as

$$
\begin{aligned}
E_{A}= & P_{\text {out }} T_{\mathrm{s}}^{4}+\left(1-P_{\text {out }}\right) T_{\mathrm{s}} \\
& +\frac{1-P_{S}}{P_{S}}\left(T_{\mathrm{c}}+\text { DIFS }+\bar{W}\right) .
\end{aligned}
$$

Then from (3.11), the average MAC delay $\bar{D}$ is re-written as

$$
\begin{aligned}
\bar{D}= & P_{B}\left(\bar{B} / 2+\mathrm{DIFS}+\bar{W}+E_{A}\right) \\
& +\left(1-P_{B}\right)\left(\mathrm{DIFS}+W / 2+E_{A}\right) \\
= & P_{\text {out }} T_{\mathrm{s}}^{4}+\left(1-P_{\text {out }}\right) T_{\mathrm{s}}+\frac{1-P_{S}}{P_{S}}\left(T_{\mathrm{c}}+\mathrm{DIFS}+\bar{W}\right) \\
& + \text { DIFS }+P_{B}(\bar{B} / 2+\bar{W})+\left(1-P_{B}\right)(W / 2)
\end{aligned}
$$

From equation (3.16), we can get the average MAC delay of CWSP as

$$
\begin{aligned}
\overline{D^{5}}= & P_{B}^{5}\left(\overline{B^{5}} / 2+\mathrm{DIFS}+\bar{W}+E_{A}\right) \\
& +\left(1-P_{B}^{5}\right)\left(\mathrm{DIFS}+W / 2+E_{A}\right) \\
= & P_{\text {out }} T_{\mathrm{s}}^{4}+\left(1-P_{\text {out }}\right) T_{\mathrm{s}}^{5}+\frac{1-P_{S}}{P_{S}}\left(T_{\mathrm{c}}^{5}+\mathrm{DIFS}+\bar{W}\right) \\
& + \text { DIFS }+P_{B}^{5}\left(\overline{B^{5}} / 2+\bar{W}\right)+\left(1-P_{B}^{5}\right)(W / 2),(3.17
\end{aligned}
$$

and the average MAC contention delay of SWSP as

$$
\begin{aligned}
\bar{D}^{2}= & P_{B}^{2}\left(\overline{B^{2}} / 2+\mathrm{DIFS}+\bar{W}+E_{A}\right) \\
& +\left(1-P_{B}^{2}\right)\left(\mathrm{DIFS}+W / 2+E_{A}\right) \\
= & P_{\text {out }} T_{\mathrm{s}}^{4}+\left(1-P_{\text {out }}\right) T_{\mathrm{s}}^{2}+\frac{1-P_{S}}{P_{S}}\left(T_{\mathrm{c}}^{2}+\mathrm{DIFS}+\bar{W}\right) \\
& + \text { DIFS }+P_{B}^{2}\left(\overline{B^{2}} / 2+\bar{W}\right)+\left(1-P_{B}^{2}\right)(W / 2) .
\end{aligned}
$$

\subsection{Approximate Goodput}

Now we can obtain the approximate Goodput $S$ from the derived average access delay $\bar{D}$. The definition of Goodput is the amount of data bits, which excludes the PHY/PLCP header and MAC header, transmitted in an unit time. There are two types of data transmissions: Internet traffic and Intranet traffic with probabilities $P_{\text {out }}$ and $1-P_{\text {out }}$, respectively. If the transmission is the Intranet traffic, it will spend an extra channel switching time (CST) to tune to the indicated data channel and turn back to the common channel after finishing the data transmission. Thus, the Goodput of SWSP can be derived as

$$
S=\frac{\bar{m}_{r}-\left(1-P_{\text {out }}\right) T_{\mathrm{CST}}}{T(x)+\bar{D}} \times r,
$$

where $T_{\mathrm{CST}}$ denotes the CST delay, $r$ is the transmission rate and $T(x)$ is the idle period of each contention. The $T(x)$ is 


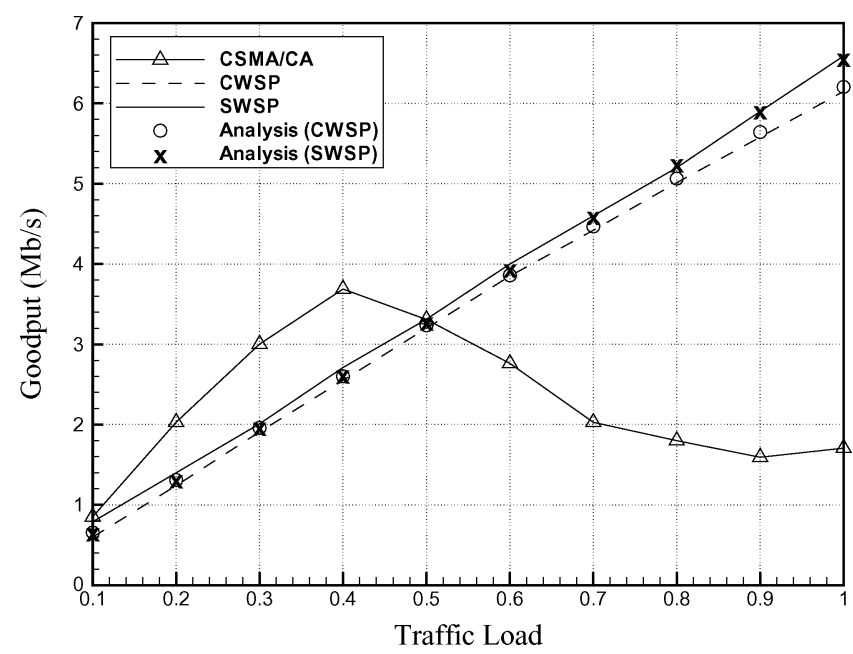

Figure 11. The comparison of Goodputs derived from analyses of the CWSP and SWSP, the simulated CWSP and the SWSP, and the CSMA/CA by varying different traffic loads, when OP $=0.1$, STAs $=10$ and $\bar{m}=55$ slots.

defined as

$$
T(x)=\left\{\begin{array}{ll}
1 / \Lambda-\bar{D}, & \text { if } 1 / \Lambda>\bar{D} \\
0, & \text { otherwise }
\end{array} .\right.
$$

Figure 11 compares the analytical Goodputs of SWSP, CWSP and the simulated Goodputs of SWSP, CWSP, and CSMA/CA at different traffic loads when $P_{\text {out }}=0.1$. The details of the simulation model will be described in the following section. From figure 11, we can see that the analytical results are very close to those of simulation experiments, which validates our proposed analytical model. Figure 11 also indicates that SWSP slightly outperforms CWSP on Goodput. Furthermore, the Goodput for CSMA/CA are higher that those for SWSP and CWSP when the network load is light. This is resulted from the overhead of the required channel switching delay (220 $\mu$ s CST delay in simulation and analysis) for SWSP and CWSP. However, when network load becomes heavy, both SWSP and CWSP outperform CSMA/CA. When the network load achieves 100\%, SWSP, CWSP and CSMA/CA achieve $6.5 \mathrm{Mb} / \mathrm{s}, 6 \mathrm{Mb} / \mathrm{s}$ and $1.6 \mathrm{Mb} / \mathrm{s}$ Goodput, respectively.

\section{Simulation models and results}

In order to evaluate the performance of our proposed WSP, two kinds of simulation models are developed to observe the Goodput of SWSP and CWSP. The Goodput is defined as the amount of pure data payload transmitted in an observed unit time and is measured in $\mathrm{Mb} / \mathrm{s}$. Simulation parameters follow the IEEE 802.11b Standard and are listed in Table 1. In our simulation models, Direct Sequence Spread Spectrum (DSSS) technique, the long Physical Layer Convergence Protocol (PLCP), and PLCP protocol data unit (PPDU) format are used.

The packet arrival rate of each mobile station follows the Poisson distribution with a mean $\lambda$, and the packet length
Table 1

System parameters in simulations.

\begin{tabular}{ll}
\hline Parameter & Normal value \\
\hline Transmission rate & $11 \mathrm{Mb} / \mathrm{s}$ \\
A slot time & $20 \mu \mathrm{s}$ \\
SIFS & $10 \mu \mathrm{s}$ \\
DIFS & $50 \mu \mathrm{s}$ \\
RTS frame length & 160 bits $(80 \mu \mathrm{s})$ \\
CTS frame length & 112 bits $(56 \mu \mathrm{s})$ \\
ACK frame length & 112 bits $(56 \mu \mathrm{s})$ \\
Number of channels & 3 \\
Preamble and PLCP header & 192 bits $(192 \mu \mathrm{s})$ \\
MAC header & 34 octets \\
CWmin & 31 slots \\
CWmax & 1023 slots \\
Average frame length & 55 slot times \\
CST delay & 11 slots $(220 \mu \mathrm{s})$ \\
\hline
\end{tabular}

is an exponential distribution with a mean of $\bar{m}$ time slots. Each mobile station maintains a FIFO waiting buffer of 64 packets. The mean packet length is set to be $1.5 \mathrm{KBytes}$ (i.e. 55 time slots at $11 \mathrm{Mb} / \mathrm{s}$ transmission rate, excluding PHY and MAC header). The network load consists of uplink (from STAs to AP) and downlink (from AP to STAs) traffic. Each simulation run lasts 100 seconds $\left(\approx 5 \times 10^{6}\right.$ time slots $)$ and each simulation result is obtained from averaging the results of 10 independent simulations.

Several assumptions are also made to reduce the complexity of the simulation model:

- All STAs support the $11 \mathrm{Mb} / \mathrm{s}$ data rate.

- All control frames are sent at $2 \mathrm{Mb} / \mathrm{s}$.

- The propagation delay is neglected.

- The channel frame error rate (FER) is assumed $0.3 \%$.

- All STAs are active (not in power-saving mode).

- There is no interference from nearby basic service sets (BSSs).

Figure 12 shows the network topology for our first simulation model. In this simulation model, nine STAs are randomly distributed in the IWLAN, and one AP is located as a centered point for coordinating these STAs. First, we consider

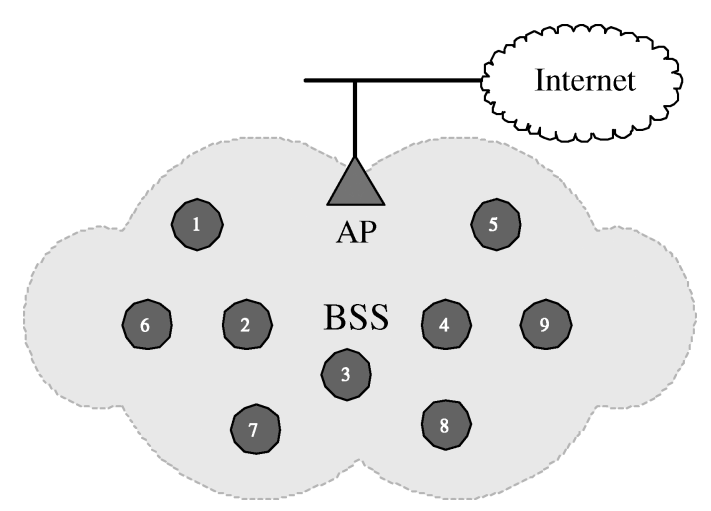

Figure 12. The network topology for our first simulation model. 


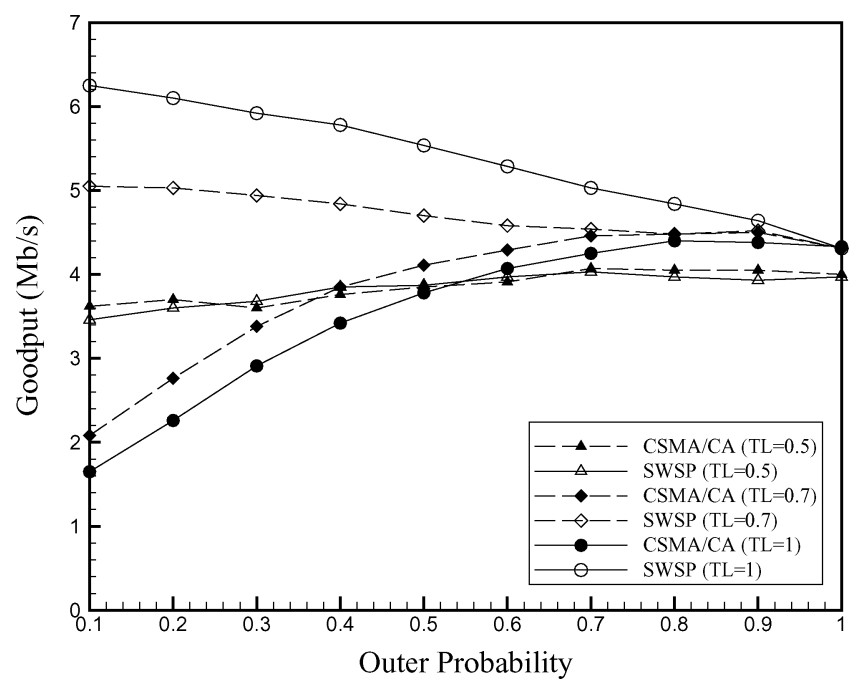

Figure 13. Comparisons of Goodputs derived from SWSP and CSMA/CA under different outer probabilities and traffic loads.

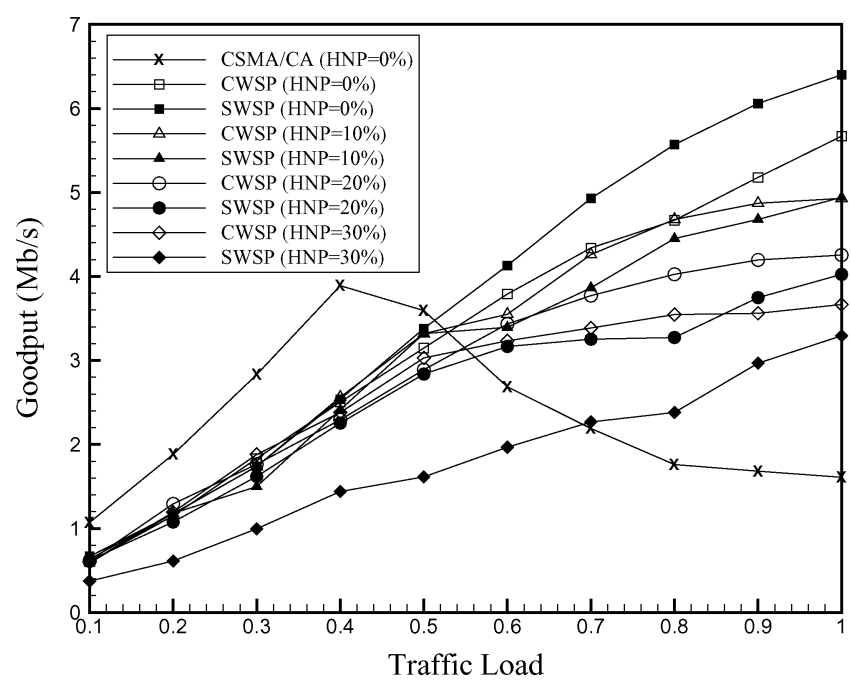

Figure 14. Comparisons of Goodputs derived from CWSP and SWSP under different hidden node probabilities and traffic loads.

the proposed SWSP in this topology and compare SWSP with IEEE 802.11b MAC protocol (named as CSMA/CA in short). Figure 13 shows the Goodput of SWSP and CSMA/CA under different outer probabilities $P_{\text {out }}$ and traffic loads (TL). We can see that the Goodput of SWSP is close to that of CSMA/CA when $P_{\text {out }}=1$ for all traffic loads under investigation. From this figure, we also observe that a higher traffic load results in a higher saturated Goodput as $P_{\text {out }}=1$. Furthermore, the Goodput of CSMA/CA is lower than that of SWSP mainly because of severe contention especially for heavy traffic load (e.g., TL = 1). In this case, 3 channels in $2.4 \mathrm{GHz}$ are fully utilized by SWSP and the network throughput gain could be up to $400 \%$. We believe that the throughput gain will become dramatically higher if the CST delay is significantly reduced (e.g., less than $100 \mu \mathrm{s}$ ) in the near future.

Then our proposed CWSP is also considered in the topology of figure 12. Figure 14 shows the Goodput of SWSP,

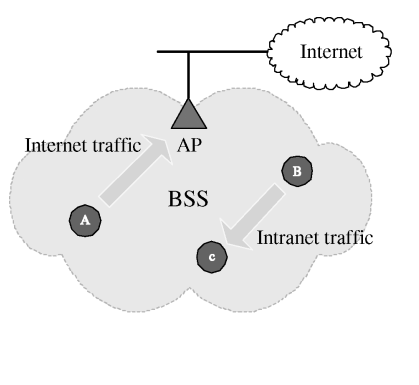

(a)

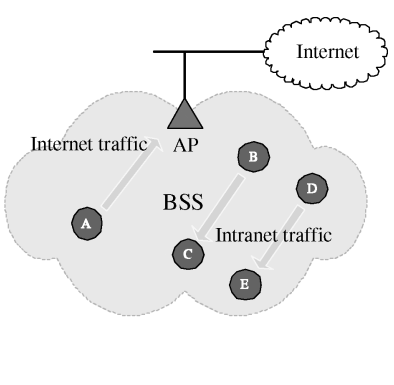

(b)
Figure 15. The second simulation model (a) The Internet traffic transmitted from STA A to AP and the Intranet traffic generated from STA B to STA C; (b) The Internet traffic transmitted from STA A to AP and the Intranet traffic transmitted between STAs $\mathbf{B}$ and $\mathbf{C}$ and between STAs $\mathbf{D}$ and $\mathbf{E}$.

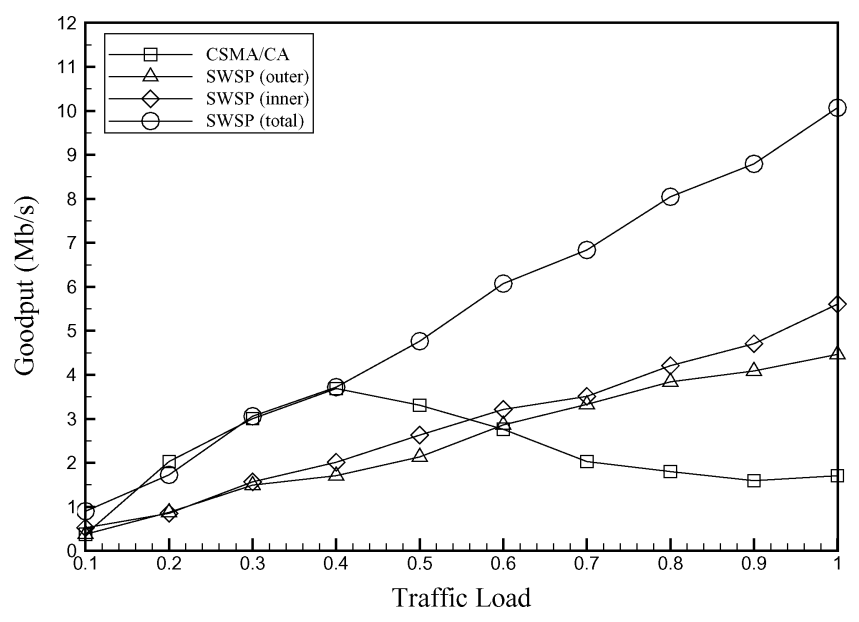

Figure 16. Comparisons of Goodputs derived from SWSP and CSMA/CA under different outer probabilities and traffic loads in scenario (a).

CWSP and CSMA/CA under different hidden node probabilities (HNP) and network TL when $P_{\text {out }}=0.1$. We can find that when HNP $=0 \%$, SWSP outperforms CWSP due to its simple handshaking procedure. However, when $\mathrm{HNP}=30 \%$, the Goodput of SWSP is lower than that of CWSP. The reason is that compared with SWSP, CWSP has better ability against the hidden-node situation. Fortunately, most wireless networks are built in home or office environment, where the hidden-node probability among STAs is very small. This implies that SWSP is a good solution for improving the network throughput in IEEE 802.11 wireless networks.

The second simulation model shown in figure 15 considers two scenarios for wireless home networks. Scenario (a) considers the network with an AP and three STAs A, B, and $\mathbf{C}$, where STA A is transmitting data packets to Internet and STA B transmits Intranet video stream to STA C. Scenario (b) is similar to Scenario (a) in addition to one more Intranet video strem transmitted between STAs D and E. The simulation result for Scenario (a) is shown in figure 16. We can find that the Goodput for the CSMA/CA protocol increases and decreases as the traffic load increases. Specifically, its Goodput approaches its maximum value (around $3.9 \mathrm{Mb} / \mathrm{s}$ ) when $\mathrm{TL}=0.4$. Unlike CSMA/CA, the overall Goodput for SWSP 


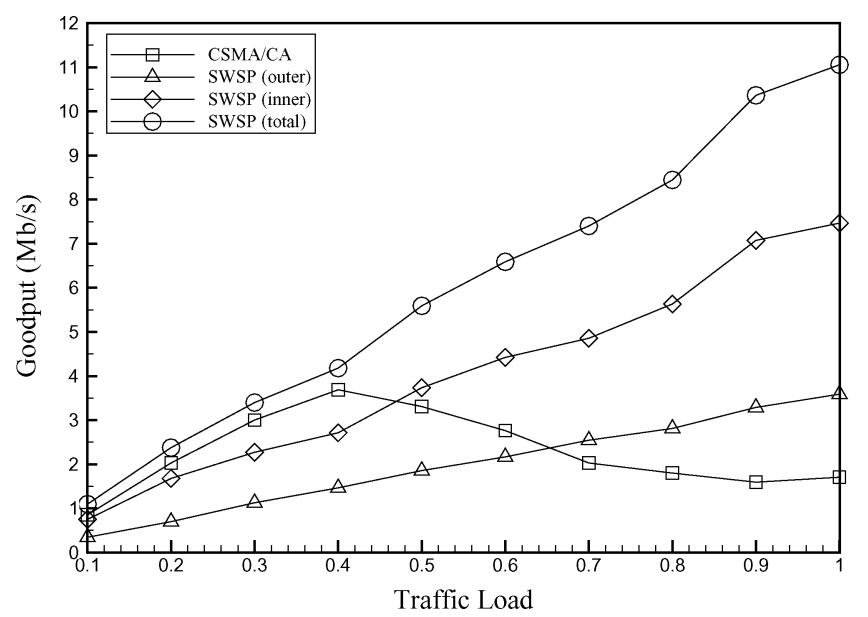

Figure 17. Comparisons of Goodputs derived from SWSP and CSMA/CA under different hidden-node probabilities and traffic loads in scenario (b).

increases proportionally with the traffic load. We also note that the Goodput for Internet traffic (outer curve) is almost the same as that for Intranet traffic (inner curve). This is because that SWSP switches Intranet traffic to another data channel and thus Internet traffic can be successfully transmitted in the common channel. When TL $=1.0$, the obtained Goodput of SWSP can be up to $10 \mathrm{Mb} / \mathrm{s}$.

Figure 17 shows the simulation result of figure 15(b). As expected, the total Goodput of SWSP is linearly proportional to TL and a higher inner curve is obtained than that in figure 16. The reason is that additional Intranet video stream transmitted between STAs $\mathbf{D}$ and $\mathbf{E}$ raise the channel utilization. Therefore we can see that the inner traffic of figure 17 obtains better Goodput than that of figure 16 and achieves the maximum Goodput around $7 \mathrm{Mb} / \mathrm{s}$ when $\mathrm{TL}=1$. We also note that the maximum Goodput for the outer curve is lower than that in figure 16 since more contentions are generated in the common channel.

\section{Conclusions}

In this paper, we pointed out the bandwidth wastage in IEEE 802.11 conventional infrastructure WLANs where one AP with one channel is used for covering all service area. In order to fully utilize all channel capacities defined in PHY specification, we proposed wireless switch protocol (WSP) based on the CSMA/CA protocol with RTS/CTS handshaking mechanism. In WSP, a pair of neighboring STAs in the same IWLAN are permitted to exchange data in another data channel different from the channel used by AP. This approach not only practically increases the channel capacity but also efficiently minimizes channel contentions. Simulations and analyses demonstrated that the proposed WSP significantly outperforms the standard CSMA/CA protocol, and the throughout gain can be even up to $400 \%$ compared to the standard CSMA/CA protocol.

\section{Acknowledgment}

This work was supported by the National Science Council, Taiwan, R.O.C., under Contract NSC92-2213-E-182-024.

\section{References}

[1] M. Ajmone-Marsan and D. Roffinella, Multichannel local area network protocols, IEEE J. Select. Areas Commun. 1, (1983) 885-897.

[2] G. Bianchi, Performance analysis of the IEEE 802.11 distributed coordination function, IEEE J. Select. Areas Commun. 18(3) (Mar. 2000) 535-547.

[3] F. Cali, M. Conti, and E. Gregori, Dynamic tuning of the IEEE 802.11 protocol to achieve a theoretical throughput limit, IEEE/ACM Trans. Networking 8(6) (Dec. 2000) 785-799.

[4] H.S. Chhaya and S. Gupta, Performance modeling of asynchronous data transfer methods in the IEEE 802.11 MAC protocol, ACM/Baltzer Wireless Networks 3(3) (Aug. 1997) 217-234.

[5] A. Chockalingam, W. Xu, M. Zorzi, and L.B. Milstein, Throughputdelay analysis of a multichannel wireless access protocol, IEEE Trans. Veh. Technol. 49(2) (Mar. 2000) 661-671.

[6] D.J. Deng and R.S. Chang, A nonpreemptive priority-based access control scheme for broadband ad hoc wireless ATM local area networks, IEEE J. Select. Areas Commun., 18(9) (Sept. 2000) 1731-1739.

[7] C.L. Fullmer and J.J. Garcia-Luna-Aceves, Complete single-channel solutions to hidden terminal problems in wireless LANs, in: Proc. IEEE ICC'97 2 (1997) 575-579.

[8] IEEE 802.11 Working Group, Part 11: Wireless LAN medium access control (MAC) and physical layer (PHY) specifications, ANSI/IEEE Std. 802.11, Sept. 1999.

[9] IEEE 802.11 Working Group, Part 11: Wireless LAN medium access control (MAC) and physical layer (PHY) specifications: Higher-speed physical layer extension in the $2.4 \mathrm{GHz}$ band, ANSI/IEEE Std 802.11, Sept. 1999.

[10] IEEE 802.11 Working Group, Part 11: Wireless LAN medium access control (MAC) and physical layer (PHY) specications: Medium access control (MAC) Enhancements for quality of service (QoS), ANSI/IEEE Std. 802.11e/D2.0, Nov. 2001.

[11] IEEE 802.11 Working Group, Part 11: Wireless LAN medium access control (MAC) and physical layer (PHY) specifications: Further higherspeed physical layer extension in the $2.4 \mathrm{GHz}$ band, ANSI/IEEE Std. 802.11g/D8.2, Jun. 2003.

[12] G.E. Keiser, Local Area Networks (McGraw-Hill, New York, 1989).

[13] Micro Linear Corporation, ML2712 2.4 GHz RF transceiver datasheet, Aug. 2001. http://www.microlinear.com

[14] B. O'Hara and A. Petrick, The IEEE 802.11 Handbook: A Designer's Companion (IEEE press, Dec. 1999).

[15] P. Z. Peebles, Probability, Random Variables and Random Signal Principles, 2nd ed. (McGraw-Hill, New York, 1987).

[16] T.G. Robertazzi, Computer Networks and Systems, Queuing Theory and Performance Evaluation, 2nd ed. (Springer-Verlag, New York, 1994).

[17] S.-T. Sheu, Y.-H. Lee, and M.-H. Chen, Providing multiple data rates in infrastructure wireless networks, in: Proc. IEEE GLOBCOM'2001, San Antonio, 3 (Nov. 2001) 1908-1912.

[18] S.-T. Sheu, Y. Tsai, and J. Chen, Delay-oriented routing protocol for wireless ad hoc networks, IEICE Trans. Commun., E84-B(6) (June 2001) 1581-1587.

[19] S.-T. Sheu and T.-F. Sheu, A bandwidth allocation/sharing/extension protocol for multimedia over IEEE 802.11 ad hoc wireless LANs, IEEE J. Select. Areas Commun. 19(10) (Oct. 2001) 2065-2080.

[20] S.-T. Sheu, Y. Tsai, and J. Chen, $\mathrm{MR}^{2}$ RP: The multi-rate and multirange routing protocol for IEEE 802.11 ad hoc wireless networks, ACM/Kluwer Wireless Networks (WINET), 9(2) (March 2003) 165177. 
[21] S.-T. Sheu, T. Chen, J. Chen, and F. Ye, The impact of RTS threshold on IEEE 802.11 MAC protocol, in: Proc. the 9th Int'l Conf. Parallel Distrib. Syst. (ICPADS'02) (Jung-Li, Taiwan, Dec. 2002) pp. 267272.

[22] F.A. Tobagi and L. Kleinrock, Packet switching in radio channels: Part II-The hidden terminal problem in carrier sense multiple-access and the busy-tone solution, IEEE Trans. Commun. COM-23(12) (Dec. 1975) 1417-1433

[23] Y.-C. Tseng, S.-L. Wu, C.-Y. Lin, and J.-P. Sheu, A multi-channel MAC protocol with power control for multi-hop mobile ad hoc networks, in: Proc. Int. Conf. DCS 2001 Workshop (2001) pp. 419-424.

[24] S.-L. Wu, C.-Y. Lin, Y.-C. Tseng, and J.-P. Sheu, A new multi-channel MAC protocol with on-demand channel assignment for multi-hop mobile ad hoc networks, in: Proc. I-SPAN 2000 (2000) pp. 232-237.

[25] Z. Zhang and Y.-J. Liu, Comments on the effect of capture on performance of multichannel slotted ALOHA systems, IEEE Trans. Commun., 41(10) (Oct. 1993) 1433-1435.

Jenhui Chen was born on October 12, 1971 in Taipei, Taiwan, Republic of China. He received the Bachelor's and Ph.D. degree in Computer Science and Information Engineering (CSIE) from Tamkang University in 1998 and 2003, respectively. In the Spring of 2003, he joined the faculty of Computer Science and Information Engineering Department at Chang Gung University and served as the Assistant Professor. He occupies the supervisor of Network Department in the Information Center, Chang Gung University. Dr. Chen once served the reviewer of IEEE Transactions on Wireless Communications, ACM/Kluwer Mobile Networks and Applications (MONET), and Journal of Information Science and Engineering. His main research interests include design, analysis, and implementation of communication and network protocols, wireless networks, milibots, and artificial intelligence. He is a member of ACM and IEEE.

E-mail: jhchen@mail.cgu.edu.tw
Ai-Chun Pang was born in Hsinchu, Taiwan, R.O.C., in 1973. She received the B.S., M.S. and Ph.D. degrees in Computer Science and Information Engineering from National Chiao Tung University (NCTU) in 1996, 1998 and 2002, respectively. She joined the Department of Computer Science and Information Engineering, National Taiwan University (NTU), Taipei, Taiwan, as an Assistant Professor in 2002. Her research interests include design and analysis of personal communications services network, mobile computing, voice over IP, and performance modeling.

E-mail: acpang@csie.ntu.edu.tw

Shiann-Tsong Sheu received his B.S. degree in Applied Mathematics from National Chung Hsing University in 1990, and obtained his Ph.D. degree in Computer Science from National Tsing Hua University in May of 1995. From 1995 to 2002, he was an Associate Professor at the Department of Electrical Engineering, Tamkang University. Since Feb. 2002, he has become a Professor at the Department of Electrical Engineering, Tamkang University. Dr. Sheu received the outstanding young researcher award by the IEEE Communication Society Asia Pacific Board in 2002. His research interests include next-generation wireless communication, WDM networks and intelligent control algorithms.

E-mail: stsheu@ee.tku.edu.tw

Hsueh-Wen Tseng received his B.S. degree in electrical engineering from Tamkang University, Taipei country, Taiwan, in 2001 and M.S. degree in electrical engineering from National Taiwan University of Science and Technology, Taipei, Taiwan, in 2003. He is currently pursuing the $\mathrm{Ph}$. D. degree at the Department of Computer Science and Information Engineering, National Taiwan University, Taipei, Taiwan. His research interests include design, analysis and implementation of network protocols and wireless communications. E-mail: hsuen@voip.csie.ntu.edu.tw 